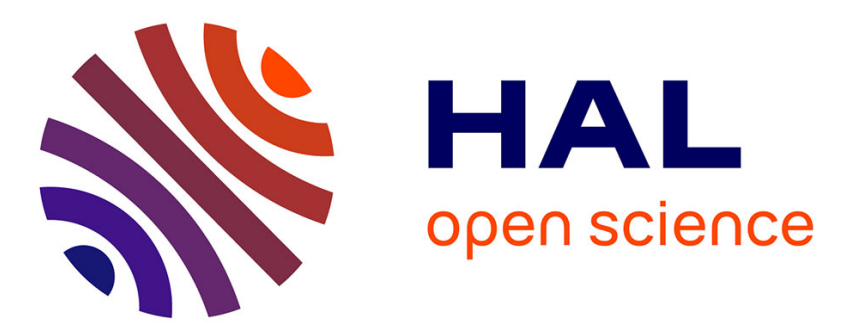

\title{
A Generalized Nash Equilibrium analysis of the interaction between a peer-to-peer financial market and the distribution grid
}

\author{
Ilia Shilov, Hélène Le Cadre, Ana Bušić
}

\section{- To cite this version:}

Ilia Shilov, Hélène Le Cadre, Ana Bušić. A Generalized Nash Equilibrium analysis of the interaction between a peer-to-peer financial market and the distribution grid. IEEE Smart Grid Comm 2021 IEEE International Conference on Communications, Control, and Computing Technologies for Smart Grids, Oct 2021, Aachen, Germany. hal-03359495

\section{HAL Id: hal-03359495 \\ https://hal.science/hal-03359495}

Submitted on 30 Sep 2021

HAL is a multi-disciplinary open access archive for the deposit and dissemination of scientific research documents, whether they are published or not. The documents may come from teaching and research institutions in France or abroad, or from public or private research centers.
L'archive ouverte pluridisciplinaire HAL, est destinée au dépôt et à la diffusion de documents scientifiques de niveau recherche, publiés ou non, émanant des établissements d'enseignement et de recherche français ou étrangers, des laboratoires publics ou privés. 


\section{A Generalized Nash Equilibrium analysis of the interaction between a peer-to-peer financial market and the distribution grid}

\author{
Ilia Shilov $1,2, *$
}

\author{
Hélène Le Cadre ${ }^{2}$
}

\author{
Ana Bušić ${ }^{1}$
}

and provide an insight on impact on the market (efficiency, individual rationality, incentive compatibility), and regulatory properties (transparency, fairness, etc.), and computational cost.

Practical problem for peer-to-peer implementation is related to the feasibility of the power flows corresponding to the bilateral trades negotiated on the financial market, regarding distribution grid network constraints. In case of infeasibility, some trades might be curtailed and the resulting loss allocated to the agents. Another important aspect which justifies the need for financial and physical level decoupling is the information sharing between prosumers and the DSO, as the latter might be reluctant to share the sensitive power grid related information with the former. This research topic has been addressed by several studies, e.g. [1] proposes a methodology to assess the impact of peer-to-peer transactions on the physical network and ensures that the physical network constraints are not violated. [11], [10] investigate multi-agent simulation framework and a consensus-based approach for peer-to-peer electricity trading in a microgrid respectively. Cooperative or noncooperative Stackelberg games are studied in [12], [13], with an assumption that the distribution system operator (DSO) acts as the leader and prosumers, as followers.

In our model we focus on the interaction between (i) the financial level, in which the agents minimize the sum of their generation flexibility cost and bilateral trading costs minus their usage benefit, and (ii) the physical level, in which the DSO minimizes the total generation flexibility cost taking into account the physics of the distribution network, which we model through a linear DC power-flow approximation. The interactions between the two levels are modeled as generalized Nash equilibrium problems (GNEP), i.e., noncooperative games which endogenized shared coupling constraints within the agents' parametrized optimization problems. We analyze generalized Nash equilibrium (GNE) [2], [9], and a refinement of it, called variational equilibria (VE) [2], assuming that the shadow variables associated with the shared coupling constraints are aligned among the agents.

Our paper key contributions can be summarized as follows: (1) We formally define the optimization problems for financial market and DSO, and formulate the interaction between the financial and physical levels as a GNEP (see Fig. 1, inspiration from [8]). We consider a two-player GNEP, in which the financial level is operated in a centralized fashion by a Market Operator (MO). We compare the two-player GNEP outcome
1 Inria Paris, DI ENS, CNRS, PSL University, France

2 VITO/EnergyVille, Thorpark 8310, Genk, Belgium

* Corresponding author, email: ilia.shilov@inria.fr 




Fig. 1: Simplified example of the two-level interaction

to a N+1 GNEP outcome, in which the financial level made of $\mathrm{N}$ prosumers is operated in a fully distributed peer-to-peer fashion to the GNEP in which the financial level is operated in a fully distributed peer-to-peer fashion. We show that the resulting GNEs are Pareto efficient under certain conditions. (2) We characterize the solution of the GNEPs and discuss the effects of the prosumers' pricing mechanism, which captures the interaction between the financial and physical levels. We provide an illustrative example that demonstrates that in case the two levels are uncoupled, there might be a free-lunch behaviors, i.e. agents increasing their trades up to infinity to minimize their costs. On the contrary, we prove that there is no such possibility in our model. (3) To illustrate our results, we show that our problem is a generalized potential game (GPG) and implement the Gauss-Seidel best response algorithm, which converges to a solution of the two-level game. In addition, we investigate the impact of RES-based generation on the market social cost and DSO's activation cost under the different levels of the generation flexibility and flexible demand available. We also consider the impact of functional dependence of the congestion cost term in the agents' trading costs functions, on the market social cost.

\section{A. Notations}

Bold symbol $\boldsymbol{x}$ denotes a vector and capital italic symbol $\mathcal{X}$ denotes a set. Exclusion of a set is denoted by $\mathcal{X} \backslash \mathcal{A}:=$ $\{x \mid x \in \mathcal{X}, x \notin \mathcal{A}\} . x \perp y$, means $x \geq 0, y \geq 0$ and $x y=0$. $\Pi_{n}$ denotes a cost function of agent $n$. To make a presentation concise, we sometimes omit the dependence of $\Pi_{n}$ on the decision variables.

\section{DESCRIPTION}

We consider a single-settlement market for energy trading made of a set $\mathcal{N}$ of $N$ agents (prosumers) - each one of them being located in a node of the distribution grid. On top of the physical level network, the agents form a trading network which is modeled as a connected undirected graph $G^{M}:=\left(\mathcal{N}, E^{M}\right)$ where $E^{M} \subseteq \mathcal{N} \times \mathcal{N}$ is the set of trading links between the players, which reflects the financial level network structure. We denote $\Gamma_{n}$ to be the set of neighbors of $n$ in this trading network, that reflects the agents she wants to trade with. In this financial level agents make the decisions about their demand $D_{n}$, generation flexibility $G_{n}$ and bilateral financial trades $q_{n m} \forall m \in \Gamma_{n} \backslash\{n\}$. If $q_{n m} \geq 0$, then $n$ buys $q_{n m}$ from $m$, otherwise $\left(q_{n m}<0\right) n$ sells $-q_{n m}$ to $m$. Inequality means that we allow for the surplus during the electricity trading. The surplus is handled by an aggregator, who can sell it on the wholesale market. The modelling of the aggregator's optimization problem is out of the scope of the current work.

On the physical level, we consider a distribution grid, which is represented by an undirected graph $G^{D S O}:=\left(\mathcal{N}, E^{D S O}\right)$, where $E^{D S O} \subseteq \mathcal{N} \times \mathcal{N}$ is a set of the distribution lines between agents. Let $\Omega_{n}$ be the set of the agents with whom agent $n$ is connected in the distribution grid (note that $\Omega_{n}$ does not necessary coincide with $\Gamma_{n}$ ). DSO makes a decision about power flows $F_{n m}$, voltage angles $\theta_{n}$ and coefficient $\rho_{n}$ for the fraction of the generation flexibility to be used.

To model the interaction between the two levels, we assume that the decision variables $D_{n}$ and $G_{n}$ of the agents act as the parameters in the DSO optimization problem. DSO's decision variable $\rho_{n}$ and the Lagrangian multiplier $\gamma_{n}$, which can be interpreted as the congestion price, are used as parameters in the agent $n$ 's optimization problem. This interaction model implies that each agent $n$ chooses the level of the generation flexibility she is willing to utilize, while the DSO chooses the share of this generation flexibility to use. Supply-demand balance constraint should hold both on the financial and physical levels. Moreover, the coupling between the two levels appears explicitly through the congestion price, a function of which is a component of the bilateral trading costs of the agents.

\section{A. Financial level}

1) Feasibility sets: For each agent $n \in \mathcal{N}$, we introduce $\mathcal{D}_{n}:=\left\{D_{n} \in \mathbb{R}_{+} \mid \underline{D}_{n} \leq D_{n} \leq \bar{D}_{n}\right\}$ as agent $n$ 's demand set, with $\underline{D}_{n}$ and $\bar{D}_{n}$ being the lower and upper-bounds on demand capacity and $G_{n}:=\left\{G_{n} \in \mathbb{R}_{+} \mid \underline{G}_{n} \leq G_{n} \leq \bar{G}_{n}\right\}$ be agent n's generation flexibility set, where $\underline{G}_{n}$ and $\bar{G}_{n}$ are the lower and upper-bounds on activation capacity. Let $\Delta G_{n}$ denote the RES-based generation at node $n$.

We impose an inequality on the trading reciprocity:

$$
q_{n m}+q_{m n} \leq 0
$$

which couples agents' bilateral trading decisions ${ }^{1}$. We denote $\zeta_{n m}$ the corresponding dual variable. It means that, in the case where $q_{n m}>0$, the quantity that $n$ buys from $m$ can not be larger than the quantity $q_{m n}$ that $m$ is willing to offer to $n$.

Local supply and demand balance leads to the following equality in each node $n$ in $\mathcal{N}$ :

$$
D_{n}=G_{n}+\Delta G_{n}+\sum_{m \in \Gamma_{n}} q_{m n}=G_{n}+\Delta G_{n}+Q_{n},
$$

\footnotetext{
${ }^{1}$ Inequality means that there might be an energy surplus in the system. Surplus might be handled by a third party player (aggregator) who is part of the game and who would compensate the consumers for the energy surpluses.
} 
where $Q_{n}$ is defined as the net import at node n. Corresponding dual variable is denoted as $\lambda_{n}$

2) Objective function: In each node $n$ we model the generation flexibility cost as a quadratic function of local activated flexibility, using three positive parameters $a_{n}, b_{n}$ and $d_{n}$ :

$$
C_{n}^{G}\left(\rho_{n} G_{n}\right)=\frac{1}{2} a_{n}\left(\rho_{n} G_{n}\right)^{2}+b_{n} \rho_{n} G_{n}+d_{n},
$$

where $\rho_{n} \in[0,1]$ is a decision variable of the DSO, which represents the the fraction of the flexibility offered by agent $n$ that is activated by the DSO.

The usage benefit perceived by agent $n$ is modeled as a strictly concave function of node $n$ demand, using two positive parameters $\tilde{a}_{n}, \tilde{b}_{n}$ and a target demand $D_{n}^{*}$, defined exogenously for agent $n$ :

$$
U_{n}\left(D_{n}\right)=-\tilde{a}_{n}\left(D_{n}-D_{n}^{*}\right)^{2}+\tilde{b}_{n}
$$

The total trading cost function of agent $n$ is denoted by:

$$
\tilde{C}_{n}\left(\boldsymbol{q}_{n}\right)=\sum_{m \in \Gamma_{n}, m \neq n} q_{n m}\left(c_{n m}+f\left(\gamma_{n}\right)\right),
$$

where parameters $c_{m n}>0$ can model taxes for energy trading or agents' preferences regarding trade characteristics [3]. In real systems, DSO does not reveal $\gamma_{n}$ explicitly, but some function $f\left(\gamma_{n}\right)$ as a function of congestion price, computed by the DSO.

Then, we write prosumer $n$ 's cost function as follows:

$$
\Pi_{n}=C_{n}^{G}\left(\rho_{n} G_{n}\right)+\tilde{C}_{n}\left(\boldsymbol{q}_{n}\right)-U_{n}\left(D_{n}\right)
$$

\section{B. Physical level}

On the physical, the DSO solves the Optimal Power Flow (OPF) problem. The original power flow equations for $\mathrm{AC}$ systems are non-linear equations of complex numbers, having a quadratic relationship between power and voltage, bringing non-convexity to the problem. We use the DC-OPF linearization of the original problem, which is classical in the OPF literature. DC-OPF formulation is used to represent distribution grids when it is important to obtain the analytical results and interpretation of the dual variables corresponding to the different prices in the electricity market [6], [7].

1) Constraints: In DC-OPF approximation, power flow on the line $\mathrm{nm}$ can be expressed as

$$
F_{n m}=\frac{1}{x_{n m}}\left(\theta_{n}-\theta_{m}\right),
$$

with the dual variable $\tau_{n m}$ associated to it, where $x_{n m}$ is the line reactance. We include the upper and lower bounds $\underline{F}_{n m} \leq F_{n m} \leq \bar{F}_{n m}$ for which we use dual variables $\underline{\phi}_{n m}, \bar{\phi}_{n m}$ correspondingly. In order to approximate the angles, we impose limits on the angle difference between connected buses:

$$
-\frac{\pi}{3} \leq \theta_{n}-\theta_{m} \leq \frac{\pi}{3}
$$

with the corresponding dual variables $\underline{\alpha}_{n}, \bar{\alpha}_{n}$. For each node, DSO ensures that local supply and demand balance holds:

$$
D_{n}=\rho_{n} G_{n}+\Delta G_{n}+\sum_{m \in \Omega_{n}} F_{n m}
$$

with $\gamma_{n}$ as a dual variable.

2) Optimization problem: Denote the joint strategy vector for the market level decision variables as $\boldsymbol{s}_{M O}:=$ $\left(s_{n}\right)_{1}^{N}$. We denote feasibility set for a DSO operator as $\mathcal{S}_{D S O}\left(\boldsymbol{s}_{M O}\right):=\left\{\boldsymbol{s}_{D S O}=\left(\rho_{n}, \theta_{n}, \boldsymbol{F}_{n}\right)_{1, \ldots, N} \mid \underline{F}_{n m} \leq F_{n m} \leq\right.$ $\bar{F}_{n m},(7),(8),(9)$ hold $\left.\forall n \in \mathcal{N}\right\}$. The DSO takes demand $D_{n}$ as the parameter and minimizes the sum of generation flexibility costs $C_{n}^{G}\left(\rho_{n} G_{n}\right)$ subject to power flow equations (7), node balance (9) and upper and lower bounds on angles and power flows constraints:

$$
\begin{array}{lll}
\min _{\rho_{n} \in[0,1], \theta_{n}, F_{n m}} & \Pi_{D S O}:=\sum_{n} C_{n}^{G}\left(\rho_{n} G_{n}\right) \\
\text { s.t. } & \boldsymbol{s}_{D S O} \in \mathcal{S}_{D S O}\left(\boldsymbol{s}_{M O}\right)
\end{array}
$$

\section{MARKET DESIGNS}

\section{A. Centralized financial market operation}

We consider two designs of the financial market level, a centralized and a decentralized (peer-to-peer) ones. Under centralized market design, the, where global Market Operator minimizes social cost of the agents on the financial level. We denote the feasibility set of agent $n$ as $\mathcal{S}_{n}\left(\boldsymbol{s}_{-n}\right):=\left\{\boldsymbol{s}_{n}=\right.$ $\left(D_{n}, G_{n}, \boldsymbol{q}_{n}\right) \mid D_{n} \in \mathcal{D}_{n}, G_{n} \in \mathcal{G}_{n},(1),(2)$ hold $\left.\forall n \in \mathcal{N}\right\}$, where $s_{-n}$ denotes is a vector that contains the concatenation of all the agents' actions excluding agent $n$.

$$
\begin{array}{ll}
\min _{G_{n}, D_{n}, q_{n m}} & \Pi_{M O}:=\sum_{n} \Pi_{n} \\
& \text { s.t. } \quad \boldsymbol{s}_{M O} \in \mathcal{S}_{M O}:=\prod_{n} \mathcal{S}_{-n}\left(\boldsymbol{s}_{-n}\right)
\end{array}
$$

We formulate the interaction between DSO and a local MO as a two-player generalized Nash equilibrium game: $\mathcal{G}:=\left\{I,\left(S_{i}\right)_{i \in I},(\Pi)_{i \in I}\right\}$, where $I$ is the set of agents, which in this framework is defined as $I:=\{M O, D S O\}$, for each $i \in I, S_{i}$ is the strategy set and $\Pi_{i}$ is the cost function. We denote the systems of the KKT conditions for the financial and physical levels as $K K T_{M O}, K K T_{D S O}$ respectively.

\section{B. Peer-to-peer financial level market design}

In this section we consider peer-to-peer setting on the financial level, in which each agent $n \in \mathcal{N}$ selfishly optimizes her demand $\left(D_{n}\right)$, energy generation $\left(G_{n}\right)$ and bilateral trades $\left(\boldsymbol{q}_{n}\right)$ with other agents in her neighborhood under constraints on demand, generation and trading capacity so as to minimize her costs. Formally, each agent in node $n \in \mathcal{N}$ solves:

$$
\begin{aligned}
\min _{D_{n}, G_{n}, \boldsymbol{q}_{n}} & \Pi_{n}, \\
\text { s.t. } & \boldsymbol{s}_{n}=\left(D_{n}, G_{n}, \boldsymbol{q}_{n}\right) \in \mathcal{S}_{n}\left(\boldsymbol{s}_{-n}\right)
\end{aligned}
$$

We formulate the interaction between the DSO and the agents as an $N+1$-player generalized Nash equilibrium game: $\mathcal{G}_{p}:=\left\{I,\left(S_{i}\right)_{i \in I},(\Pi)_{i \in I}\right\}$, where $I$ is the set of agents, which in this framework is defined as $I:=\mathcal{N} \bigcup\{D S O\}$, for each $i \in I, S_{i}$ is the strategy set and $\Pi_{i}$ is the cost function of agent $i$. We consider the KKT conditions of the game as a system given by $K K T_{p}$ which is the concatenation of $K K T_{D S O}$ and 
$K K T_{n}$ for all $n=1 \ldots N$, where $K K T_{n}$ denotes the KKT conditions for (12).

\section{EQUILIBRIUM ANALYSIS}

In our analysis we rely on Generalized Nash Equilibria and Variational Equilibria; both of them exist under mild conditions [2], [9].

Definition 1 ( [2]): A Generalized Nash Equilibrium (GNE) of the game $\mathcal{G}\left(\mathcal{G}_{p}\right)$ with coupling constraints, is a vector $\left(x_{M O}, x_{D S O}\right)$ that solves the system given by $K K T_{M O} \mathrm{~V}$ $K K T_{D S O}\left(K K T_{p}\right)$.

Definition 2: We say that GNE of $\mathcal{G}_{p}$ is induced by the Variational Equilibrium (VE) of the financial level problem if it is a $\mathrm{GNE}$ of $\mathcal{G}_{p}$ s.t.

$$
\zeta_{n m}=\zeta_{m n}, \quad \forall n \in \mathcal{N}, \forall m \in \Gamma_{n}
$$

We denote such euilibria as $G N E_{V E}$.

As it was stated above, $\zeta_{n m}$ for $n \in \mathcal{N}, \forall m \in \Gamma_{n}$ can be interpreted as bilateral energy trading prices [3]. In general, $\zeta_{n m} \neq \zeta_{m n}$, thus leading to non-symmetric energy trading prices between couple of agents. Relying on VE as solution concepts enforces a natural symmetry in the bilateral energy price evaluation between any couple of agents [3].

We note that $\left(s_{1}, \ldots, s_{n}\right)$ that solve $\vee_{n=1}^{N} K K T_{n}$ are defined by exactly the same KKT system as the social cost minimizer of the market level problem - $K K T_{M O}$. Therefore, we obtain the following result:

Proposition 3: GNE given in two-player game $\mathcal{G}$ coincides with $G N E_{V E}$ of $\mathcal{G}_{p}$.

1) Pareto-efficiency of GNE: A strategy is a Pareto efficient outcome if no joint strategy is both a weakly better outcome for all players and a strictly better outcome for some player. Formally, if $S_{G N E}$ denotes the set of the joint equilibrium strategies, then $s \in S_{G N E}$ is Pareto efficient if $\nexists \boldsymbol{s}^{\prime} \in S_{G N E}$ s.t.

$$
\forall i \in I: \Pi_{i}\left(s^{\prime}\right) \leq \Pi_{i}(s), \quad \exists i \in I: \Pi_{i}\left(s^{\prime}\right)<\Pi_{i}(s)
$$

Proposition 4: If the coefficients $c_{n m}$ in the trading costs $\tilde{C}_{n}\left(\boldsymbol{q}_{n}\right)$ of the agents are homogeneous, i.e. $c_{n m}=$ $c_{n^{\prime} m^{\prime}} \forall n, m, n^{\prime}, m^{\prime}$, then GNE of $\mathcal{G}$ and $G N E_{V E}$ of $\mathcal{G}_{p}$ are Pareto-efficient.

Proof. Denote $c:=c_{n m} \forall n, m$. Then, we can rewrite the trading cost of the agents using the supply-demand balance equality:

$$
\tilde{C}_{n}\left(\boldsymbol{q}_{n}\right)=c Q_{n}=c\left(D_{n}-G_{n}-\Delta G_{n}\right),
$$

thus the objective function $\Pi_{n}$ doesn't depend on $\boldsymbol{q}_{n}$. Note that $\Pi_{n}$ is strictly convex in $D_{n}, G_{n}$ and the feasibility set of the financial level optimization problem is convex. Then, solution of the financial level problem given by the $K K T_{M O}$ (or the $\vee_{1}^{N} K K T_{n}$ s.t. $\zeta_{n m}$ are equal) is unique w.r.t. $D_{n}, G_{n}$. Now we consider the strategies of the DSO. Objective of the DSO doesn't depend on $\theta_{n}$ and $F_{n m}$ is strictly convex in $\rho_{n}$. The feasible set is convex. Thus, we have a unique $\rho_{n}$ that solves the KKT conditions. It follows that there's no other GNE that can decrease the costs of the agents.
2) Pricing: In this section we focus on the Lagrangian multipliers that can be interpreted a market prices. From $K K T_{D S O}$ conditions, we obtain the expression for the $\gamma_{n}$ :

$$
\gamma_{n}=\bar{\phi}_{n m}-\underline{\phi}_{n m}+\left(\bar{\alpha}_{n}-\underline{\alpha}_{n}\right) x_{n m}
$$

Note that the angles $\theta_{n}, \theta_{m}$ unambiguously define $F_{n m}$, thus, for the pair of the agents $n, m$ there can be only one active constraint out of the power flow bounds $\underline{F}_{n m} \leq F_{n m} \leq \bar{F}_{n m}$ and the angles bounds (8). It follows that the dual variable $\gamma_{n}$ represents the congestion price on the physical level. From the $K K T_{M O}$ conditions for the financial level we have that the nodal price $\lambda_{n}$ associated with the supply and demand balancing constraint in node $n$ can be expressed as the sum of the bilateral trade price $\zeta_{n}$ offered by $n$ to $m$ associated with the trading reciprocity constraint (1), the coefficient $c_{n m}$ and the function $f\left(\gamma_{n}\right)$ of congestion price $\gamma_{n}$ on the physical level:

$$
\lambda_{n}=c_{n m}+f\left(\gamma_{n}\right)+\zeta_{n m}
$$

From the balance equations (9) and (2), we have that

$$
G_{n}\left(1-\rho_{n}\right)=\sum_{m \in \Gamma_{n}} F_{n m}-\sum_{m \in \Omega_{n}} q_{n m}
$$

Since $\rho_{n} \in[0,1], 1-\rho_{n} \leq 0$ we consider three cases:

1) $G_{n} \geq 0$, then $\sum_{m \in \Gamma_{n}} q_{n m} \leq \sum_{m \in \Omega_{n}} F_{n m}$

2) $G_{n} \leq 0$, then $\sum_{m \in \Gamma_{n}} q_{n m} \geq \sum_{m \in \Omega_{n}} F_{n m}$

3) $G_{n}=0$ or $\rho_{n}=1$, then $\sum_{m \in \Gamma_{n}} q_{n m}=\sum_{m \in \Omega_{n}} F_{n m}$

If node $n$ is injecting power in the grid, i.e. $G_{n} \geq 0$, then the trading cost allocated to this agent is less than the total congestion cost $f\left(\gamma_{n}\right) \sum_{m \in \Omega_{n}} F_{n m}$ caused by this agent on the physical level and vice versa.

3) No free lunch behavior: Term $\sum_{m \in \Gamma_{n}} f\left(\gamma_{n}\right) q_{n m}$ is crucial in the trading costs of the agents. In the absence of $f\left(\gamma_{n}\right)$ in the trading costs, we might observe the free lunch behavior - situation in which the financial trades are increasing up to infinity in order to decrease the trading costs $\tilde{C}_{n}\left(\boldsymbol{q}_{n}\right)$. We illustrate this in the following example:

Example: Consider 3-node network, which is represented by a complete graph. Assume that the trading costs of the agents are given by $\tilde{C}_{n}\left(\boldsymbol{q}_{n}\right)=\sum_{n \in \Gamma_{n}} c_{n m} q_{n m}$ and the coefficients $c_{n m}$ are $\left(c_{12}, c_{13}\right)=(1,1),\left(c_{21}, c_{23}\right)=(1,3)$, $\left(c_{31}, c_{32}\right)=(2,1)$. Let $\left(\boldsymbol{q}_{1}, \boldsymbol{q}_{2}, \boldsymbol{q}_{3}\right)$ be a feasible vector of the trading decisions s.t. $q_{13}, q_{21}, q_{32}<0$ and $q_{31}, q_{12}, q_{23}>0$. Then, w.l.o.g. pick node 3 and assume that it increases amount of energy she sells to node 1: $q_{31}^{\prime}=q_{31}-\varepsilon$. Then, it also has to buy the same additional amount from node $2: q_{32}^{\prime}=q_{32}+\varepsilon$. Then, new trading cost $\tilde{C}_{3}\left(\boldsymbol{q}_{3}^{\prime}\right)=2 *\left(q_{31}-\varepsilon\right)+1 *\left(q_{32}+\varepsilon\right)=$ $\tilde{C}_{3}\left(\boldsymbol{q}_{3}\right)-\varepsilon$. Similarly, for node $2, \tilde{C}_{2}\left(\boldsymbol{q}_{2}^{\prime}\right)=\tilde{C}_{2}\left(\boldsymbol{q}_{2}\right)-2 \varepsilon$ and for node 1: $\tilde{C}_{1}\left(\boldsymbol{q}_{1}^{\prime}\right)=\tilde{C}_{1}\left(\boldsymbol{q}_{1}\right)$. Note, that all the bilateral trade constraints (1) remain feasible and that $Q_{n}$ do not change. Thus, by increasing $\varepsilon$, agents are able to decrease their costs without violating any constraint.

The following result states, that adding the term $f\left(\gamma_{n}\right) Q_{n}$ in the trading costs of the agents prohibits the free-lunch behavior. 
Proposition 5: Free lunch behavior is not possible in the $G N E_{V E}$ if the trading costs of the agents are given by (5), i.e. $\nexists \boldsymbol{s}_{n}$ s.t. $\boldsymbol{s}_{n} \in S_{n}$ and $\tilde{C}_{n} \rightarrow-\infty$.

Proof. To consider free-lunch behavior, it is sufficient to investigate the cycles in graph $G$. Consider a cycle of length $k$ : $G_{C_{k}}:=\left(\left(n_{1}, \ldots, n_{k}\right), E_{C_{k}}\right)$. We consider a part of the trading cost function of agent $i$ is that corresponds to the trades made inside this cycle:

$$
\begin{aligned}
\tilde{C}_{i}^{G_{C_{k}}}\left(\boldsymbol{q}_{i}\right) & =c_{i, i-1} q_{i, i-1}+c_{i, i+1} q_{i, i+1}+f\left(\gamma_{i}\right)\left(q_{i, i-1}+q_{i, i+1}\right) \\
& =\lambda_{i}\left(q_{i, i-1}+q_{i, i+1}\right)-\zeta_{i, i-1} q_{i, i-1}-\zeta_{i, i+1} q_{i, i+1}
\end{aligned}
$$

Assume that one agent changes her trades by adding $\varepsilon$ to the amount she buys and subtracting $\varepsilon$ from the amount she sells. In order to have free-lunch behavior, it is necessary that all the changes in the trading costs of the agents in $G_{C_{k}}$ induced by this change are non-positive with one agent having strictly decreased cost. Thus, the change in the sum of the total costs of all agents in $G_{C_{k}}$ should be negative. Note, that the first term $\lambda_{i}\left(q_{i, i-1}+q_{i, i+1}\right)$ does not change, so it is sufficient to consider the last. Taking the sum over all the nodes in $G_{C_{k}}$

$$
\begin{aligned}
& -\sum_{i \in G_{C_{k}}}\left[\zeta_{i, i-1} q_{i, i-1}+\zeta_{i, i+1} q_{i, i+1}\right]= \\
& =-\left[\zeta_{2,1} q_{2,1}+\zeta_{1,2} q_{1,2}+\ldots \zeta_{1, k} q_{1, k}+\zeta_{k, 1} q_{k, 1}\right] \\
& =-\left[\zeta_{1,2}\left(q_{2,1}+q_{1,2}\right)+\cdots+\zeta_{1, k}\left(q_{1, k}+q_{k, 1}\right)\right]=0
\end{aligned}
$$

Where the equivalence to zero follows from the complementarity conditions for the bilateral trading constraints (1). We finish the proof by noting that it contradicts the necessary condition for the free-lunch behavior.

\section{NumERICAL RESULTS}

We consider the 18-node distribution network [17], for which all the parameters and the scheme are provided in [16]. Each node is a consumer with $\bar{D}_{n}>0$ and some nodes are generators (RES or conventional), therefore producing energy that can be consumed locally to meet demand $D_{n}$ and exported to the other nodes to meet the unsatisfied demand.

We are interested in two aspects of the interaction between financial and physical levels: (i) how does the penetration of RES generation affect the efficiency and performance of the two-level noncooperative game, (ii) how does the form of the trading cost affect the efficiency of the market, more precisely, what is the effect of the different functions $f(\cdot)$ that we apply to the congestion price $\gamma_{n}$.

1) Potential form of the game: Computing GNE in the general case might be a challenging task. For a specific type of GNEP, which is called Generalized Potential Games (GPG) there are established approaches in the literature that compute both GNE [14] and VE [15]. A GNEP is a Generalized Exact Potential Game if (i) the feasible set of the game is non empty, (ii) there exists a continuous function $P(\boldsymbol{x}): \mathbb{R}^{N} \rightarrow \mathbb{R}$ such that for all $n$, for all $s_{-n}$ (such that $\mathcal{S}_{n}\left(s_{-n}\right)$ is not empty), and for all $\boldsymbol{s}_{n}, z_{n} \in \mathcal{S}_{n}\left(\boldsymbol{s}_{-n}\right)$

$$
\Pi_{n}\left(\boldsymbol{s}_{n}, \boldsymbol{s}_{-n}\right)-\Pi_{n}\left(z_{n}, \boldsymbol{s}_{-n}\right)=P\left(\boldsymbol{s}_{n}, \boldsymbol{s}_{-n}\right)-P\left(z_{n}, \boldsymbol{s}_{-n}\right)
$$

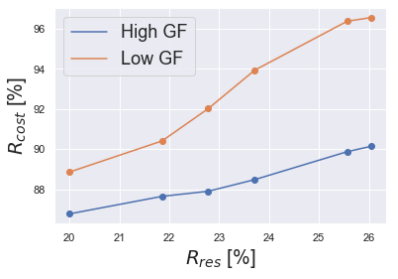

Fig. 2: Flexible generation

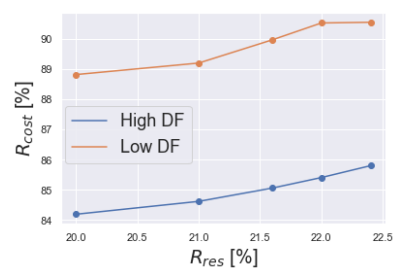

Fig. 3: Flexible demand
Proposition 6: Problem $\mathcal{G}_{p}$ is a is a Generalized Exact Potential Game (GPG) with a potential function given by

$$
P=\sum_{n}\left[C_{n}^{G}\left(\rho_{n} G_{n}\right)+\tilde{C}_{n}\left(\boldsymbol{q}_{n}\right)-U_{n}\left(D_{n}\right)\right]
$$

Proof. Indeed, the feasible set of the problem is nonempty and we can check directly that (17) holds.

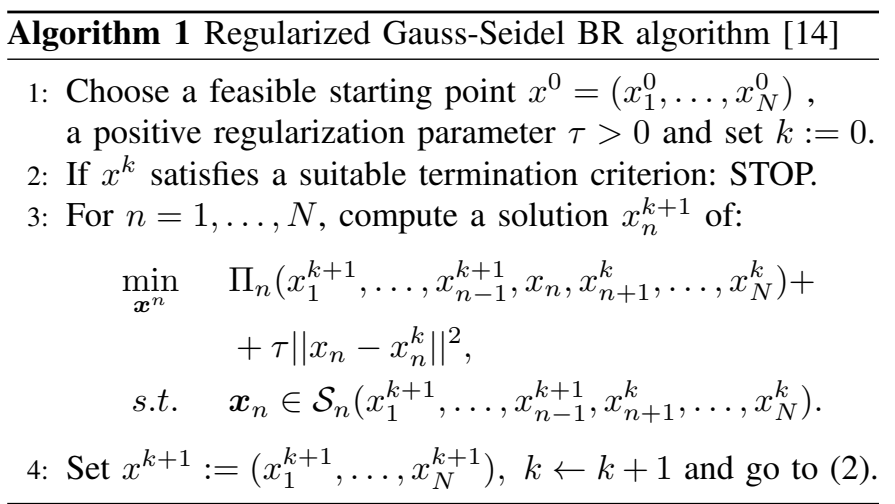

First, we investigate the efficiency loss caused by the peerto-peer equilibrium computation with respect to centralized solution. Formally, we compute the ratio of the total cost of the system computed at an optimum and the total cost of the system at a GNE:

$$
R_{\text {cost }}:=\frac{\operatorname{Cost}_{\left(\boldsymbol{s}_{1}, \ldots, \boldsymbol{s}_{N}, \boldsymbol{s}_{D S O}\right) \in S C}\left(\boldsymbol{s}_{1}, \ldots, \boldsymbol{s}_{N}, \boldsymbol{s}_{D S O}\right)}{\operatorname{Cost}_{\left(\boldsymbol{s}_{1}, \ldots, \boldsymbol{s}_{N}, \boldsymbol{s}_{D S O}\right) \in G N E}\left(\boldsymbol{s}_{1}, \ldots, \boldsymbol{s}_{N}, \boldsymbol{s}_{D S O}\right)}
$$

2) RES penetration and flexibility: Define the percentage of the RES-based generation penetration in the system as

$$
R_{r e s}=\frac{\sum_{n \in \mathcal{N}} \Delta G}{\sum_{n \in \mathcal{N}} \bar{G}_{n}}
$$

There are two important points to consider when we increase the amount of RES in the system: (i) how much does it increase the efficiency loss of the system, (ii) what is the maximum amount of RES that we can inject so that the problem remains feasible.

Figure 2 illustrates how increasing RES generation percentage in the system we can approach the social cost optimum in the different settings of the generation flexibility availability. Low GF and High GF correspond to the low and high values of the $\bar{G}_{n} \forall n \in \mathcal{N}$ respectively. On the horizontal axis we put the percentage $R_{\text {res }}$ of the RES-based generation in the network, which varies in the intervals that ensure that the 


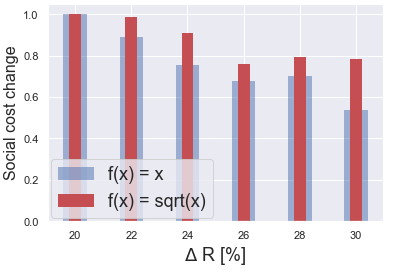

Fig. 4: Agents' cost changes

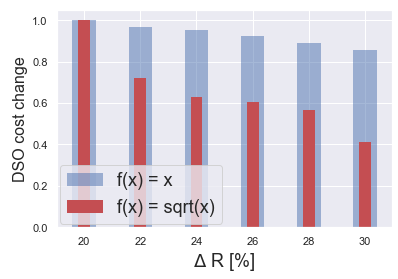

Fig. 5: DSO cost changes problem is feasible in all settings considered. For the Low GF framework, the problem becomes infeasible for $R_{\text {res }}>23 \%$ (or $R_{\text {res }}>27 \%$ ) as there is not enough flexible resource in the nodes to avoid congestion. First, note, that the efficiency of the GNE in the High GF is lower, which is caused by the bigger feasible sets, thus, the increased ability of the agents to act selfishly and deviate more from the SC optimum. Increasing the amount of RES generation available affects the Low $G F$ the most: as soon as the RES generation of the agents increases, agents obtain more freedom and are able to better adjust their generation/trades, which leads to the increase in the efficiency with a high growth rate. High $G F$ efficiency growth is much less affected by the RES injection, because the agents already have had a lot of freedom for their actions even on the low values of $R_{\text {res }}$.

Similar behavior is observed when we investigate the effects of the demand flexibility on the efficiency of the system. Figure 3 shows the changes in the efficiency for the High DF and Low $D F$ settings w.r.t. $R_{\text {res }}$. Again, more flexibility in the decisions of the agents leads to the lowest values of the efficiency of the system and lower level of the flexibility induces the highest efficiency growth rate w.r.t. $R_{\text {res }}$.

3) RES penetration and pricing functions: We investigate how the form of the function $f(\cdot)$ affects the total cost of the agents. For our experiments we choose $f(\cdot)$ to be monotonically increasing, continuous function: we take $f(x)=\sqrt{x}$ and $f(x)=x$. Figures 4 and 5 illustrate how the total costs of all agents and DSO change when we increase the RES penetration. On the $y$-axis we put the ratio between the costs at the given value of $R_{\text {res }}$, divided by the first entry, i.e. the cost at a value $R_{\text {res }}=20 \%$.

The decrease of the agents' and DSO costs shows the same tendency for both of the functions $f(x)=\sqrt{x}$ and $f(x)=x$ on Figures 4 and 5. While the decrease rate of the DSO cost is higher for $f(x)=\sqrt{x}$ it is the opposite for the agents' cost. In this example $f(x)=\sqrt{x}$ seems to be a good choice for the DSO, while bringing some disadvantages for the agents. As it is illustrated in this example, it is important to design a suitable $f(\cdot)$ which would benefit both layers of the model, thus calling for a further research.

\section{CONCLUSION}

We formulate a generalized Nash equilibrium problem which models the interaction between the financial peer-topeer electricity market level and the physical level (distribution grid) operated by the DSO. We provide characterization of the
GNE under different designs of the financial level prosumers' market. We discuss the effects of the trading cost form on the equilibria, focusing on properties such as Pareto efficiency, no free-lunch behavior and Lagrangian multipliers pricing interpretation.

As a future research direction, we will investigate formally the impact of the functions used to compute the modified nodal prices that the DSO uses to charge the prosumers. Another interesting direction is to consider the different physical layer models, e.g. second cone order programming (SOCP). It is interesting to provide the bound on the Price of Anarchy to evaluate the efficiency loss caused by the decentralization of the decisions in the financial level.

\section{REFERENCES}

[1] J. Guerrero, A. C. Chapman, and G. Verbic, "Decentralized P2P energy trading under network constraints in a low-voltage network," IEEE Transactions on Smart Grid, 2018.

[2] A. A. Kulkarni and U. V. Shanbhag, "On the variational equilibrium as a refinement of the Generalized Nash equilibrium", Automatica, vol. 48, no. 1, pp. 45-55, 2012.

[3] H. Le Cadre, P. Jacquot, C. Wan and C. Alasseur, "Peer-to-peer electricity markets: from variational to generalized Nash equilibrium", European Journal of Operational Research, vol. 282, no.2, pp. 753-771, 2020.

[4] F. Moret, P. Pinson, and A. Papakonstantinou, "Heterogeneous risk preferences in community-based electricity markets", European Journal of Operational Research, vol. 287, no. 1, pp. 36-48, 2020.

[5] W. Tushar, T. K. Saha, C. Yuen, D. Smith, and H. V. Poor, "Peer-to-Peer Trading in Electricity Networks: An Overview", IEEE Trans. on Smart Grid, vol. 11, no. 4, pp. 3185-3200, 2020

[6] S. Hanif, K. Zhang, C. M. Hackl, M. Barati, H. B. Gooi and T. Hamacher,"Decomposition and Equilibrium Achieving Distribution Locational Marginal Prices Using Trust-Region Method," in IEEE Transactions on Smart Grid, vol. 10, no. 3, pp. 3269-3281, 2019

[7] R. Li, Q. Wu and S. S. Oren, "Distribution Locational Marginal Pricing for Optimal Electric Vehicle Charging Management," in IEEE Transactions on Power Systems, vol. 29, no. 1, pp. 203-211, 2014

[8] Y. Liu, H. Zhang, W. Gong and D. Towsley, "On the interaction between overlay routing and underlay routing," Proceedings IEEE 24th Annual Joint Conference of the IEEE Computer and Communications Societies, 2005

[9] H. Yin, U. V. Shanbhag, and P. G. Mehta, "Nash Equilibrium Problems With Scaled Congestion Costs and Shared Constraints", IEEE transactions on automatic control, vol. 56, no. 7, pp. 1702-1708, 2011.

[10] Y. Zhou, J. Wu, and C. Long, "Evaluation of peer-to-peer energy sharing mechanisms based on a multiagent simulation framework," Applied Energy, vol. 222, pp. 993-1022, 2018.

[11] E. Sorin, L. Bobo, and P. Pinson, "Consensus-based approach to peer-topeer electricity markets with product differentiation," IEEE Transactions on Power Systems, vol. 34, no. 2, pp. 994-1004, 2019

[12] W. Tushar et al., "Grid Influenced Peer-to-Peer Energy Trading," in IEEE Transactions on Smart Grid, vol. 11, no. 2, pp. 1407-1418, 2020

[13] S. Maharjan, Q. Zhu, Y. Zhang, S. Gjessing, and T. Basar, "Dependable demand response management in the smart grid: A stackelberg game approach," IEEE Transactions on Smart Grid, vol. 4, no. 1, pp. 120-132, 2013.

[14] F. Facchinei, V. Piccialli, and M. Sciandrone, "Decomposition Algorithms for Generalized Potential Games", Computational Optimization and Applications 50, 2011.

[15] G. Belgioioso and S. Grammatico"A distributed proximal-point algorithm for Nash equilibrium seeking in generalized potential games with linearly coupled cost functions", 18th European Control Conference (ECC), 2019

[16] I. Shilov Github repository, https://github.com/ishilov/spider-network, 2021

[17] H. Le Cadre, I. Mezghani, A. Papavasiliou, "A game-theoretic analysis of transmission-distribution system operator coordination", European Journal of Operational Research, vol. 274, no. 1, pp. 317-339, 2019 